\title{
An analysis of individual and department triage variances to identify, quantify, and improve markers of nurse triage accuracy.
}

\section{Rebecca Cotton, Richard Drew, Matthew Douma, Domhnall O'Dochartaigh, Candice Keddie, Karen Muncaster, Christopher Picard}

Background: Canadian Emergency Departments (ED) use the five-point Canadian Triage Acuity Scale (CTAS) to sort and prioritize patients according to acuity. CTAS scores are used to make decisions on patient flow, staffing complement, and funding. Despite this, there is a paucity of literature describing how CTAS data can be audited, and how the data can inform quality improvement/assurance (QI/QA).

Implementation: Triage data downloaded from Tableau were analyzed using Microsoft Excel and IBM SPSS 26. Staff were informed of the audit using email and social media, and invited to discuss the results with educators and administrators. Staff identified for intervention were approached individually with the administrative plan. Anonymized versions of the work plan were posted on the departmental audit board. Nurses triaging greater than $50 \%$ department average were offered the option to triage less frequently, while nurses triaging less than $50 \%$ the department average were preferentially placed in triage. Nurses triaging fewer than 100 patients per year were informed they would be relieved of triage responsibility unless their rates increased above threshold. Nurses "down-triaging" patients at rates greater than 2 SD were informed that if their practice remained outside $2 \mathrm{SD}$ at repeat audit they would be relieved of triage responsibility until they voluntarily completed CTAS refresher training. Nurses with average assigned CTAS scores $>2$ SD department average had 20 visits randomly audited per month for error/appropriateness.

Evaluation Method: Computer-assisted analysis of complete triage records was conducted for August 2019 to August 2020 at the Misericordia Hospital Emergency. Complete triage entries of every patient triaged by all triage trained nurses in the department were examined. Nurse's with practice variation two deviations from department mean were identified and received additional detailed audits. Items examined for error were: FTE adjusted triage frequency; average CTAS score assigned; triage score manual override "down/up-triage" rate; proportion of absent Numeric Pain Scores (NPS) for patients with primary presenting complaints of pain; and vital signs modifier error rates. Initial department averages were used for benchmarking individual nurses; zone averages were used to benchmark department performance. Nurses were interviewed, audit results and action plans were posted. Repeat audits were performed on a threemonth basis and benchmarked to initial measures, and a staff awareness campaign was enacted to improve NPS scoring. Data were extracted using text-parsing algorithms programmed into Microsoft Excel and analyzed using IBM SPSS 26. Data were normally distributed and descriptive statistics were calculated using means and standard deviations. T-testing was used for comparisons, and all testing was two-tailed with a pre-defined significance set at 0.05 .

Results: After the $3^{\text {rd }}$ quarterly audit and associated interventions, global improvements were 
appreciated in triage nurse practice. There was a $68 \%$ reduction in the need for administrative action $(n=51, n=18)$ with reduced variance in individual nurse triage rates and a $50 \%$ reduction in nurses who triaged $>50 \%$ more patients than their peers. $50 \%$ fewer nurses had a mean triage rate $>.02$ above or below department average, there was an $86 \%$ reduction in high risk vital sign error rates, a $78 \%$ reduction in "down-triage" rates, and a $6.5 \%$ improvement in documentation of numerical pain scores.

\section{Advice and Lessons Learned}

1) Triage data analytics can rapidly identify staff with significant deviations from the average, making auditing and QI/QA activities more efficient and effective.

2) Having a concrete performance management framework and dissemination plan in place are essential for auditing to have a significant impact on triage consistency and quality over time.

3) Future QI/QA work should consider expanding computer-assisted text parsing to identify patients at risk for mis-triage for reasons other than vital sign derangement, which will allow for broader ED rollout across the Edmonton Zone and beyond. 